\title{
Creep response in shear of clayey geo-materials under saturated and unsaturated conditions
}

\author{
Nor Shahidah Mohd Nazer ${ }^{1,2, a}$ and Alessandro Tarantino ${ }^{1}$ \\ ${ }^{1}$ Department of Civil and Environmental Engineering, University of Strathclyde, Glasgow \\ ${ }^{2}$ Faculty of Science and Technology (Geology Programme), National University of Malaysia, Bangi, Selangor, Malaysia
}

\begin{abstract}
Translational slides in clays are often characterized by long-lasting intermittent movements associated with the fluctuations of pore-water pressure. Physically-based models designed to support hazard analysis of landslide movements and early warning systems require the integration of time-dependent (viscous) constitutive models for the shear displacements because landslide movements are typically controlled by the viscous behaviour of the clay geomaterial. This paper presents an investigation of the viscous response of a clay geo-material under saturated and unsaturated conditions. Creep and relaxation tests have been first carried out on saturated clay samples by means of direct shear box. To gain a conceptual understanding of the viscous response of the clay in shear, mechanical analogues were considered based on combinations of springs and dashpots. Preliminary tests on unsaturated samples were finally carried out to gain a first insight into the viscous response of the clay under unsaturated conditions.
\end{abstract}

\section{Introduction}

Landslides induced by rainfall often lead to disastrous hazard. Heavy rainfall accompanied by slope displacement may cause damage in terms of serviceability to houses, buildings, roads, as well as other man-made structures and brings danger to life. Translational slides in clay are usually intermittent and happening in long run. They exhibits long-term deformation and proper land management is therefore important in area prone to slope failure.

Early warning systems (EWS) are generally designed to forecast landslide hazard and detect hazard and risk zones. Designing simple EWS is important so as to avoid confusion and loss of time during emergencies [1]. EWS must incorporate field monitoring data and should be underpinned by properly designed mechanical models to allow for accurate projection of future movements. Understanding mechanisms that lead to failure and investigating its interplay with landslide kinematics is therefore vital.

When considering landslide's failure mechanism, relationships between pore-water pressure and rainfall become the subject of interest due to their inevitable contribution to landslide movements [2-4]. When rain water infiltrates into the ground, it causes fluctuations of pore water pressure in the upper portion of the soil profile (unsaturated zone). Seasonal fluctuations in pore water pressure cause significant decrease in soil shear strength as a result of pore pressure build up and may trigger slope movements. The Sarno landslide in town Campania, Italy is one example of landslides which cyclically mobilized by pore-water pressure fluctuations [5].

Movements of slopes along the slip surface are controlled by viscous behaviour mainly in the form of creep. Creep is causing slow slope movements over time under constant effective stress. Studies have shown that creeping slope may moves at imperceptible rate (several $\mathrm{mm}$ per year) and the movements may continue for long periods without reaching failure [2].

A large number of creep models in the literature, associated with early warning system for rainfall-induced landslides, are based on empirical models rather than physically-based models [6]. Empirical models are known to be limited to a specific boundary condition and did not provide clear conceptual understanding of landslide kinematics. Empirical models therefore offer limited application and also lack in theoretical basis [7].

Lack of creep studies in relation with unsaturated soil has raised great interest among researchers to investigate the time-dependence behaviour of unsaturated geomaterials by accounting the effects of matric suction. Among them, Lai et al. [8] established a linear relationship between suction and parameter from a model built for saturated soils and developed a creep model with consideration for suction effects.

In this research, we aim to simulate viscous response in shear of clayey geo-material using a mechanical

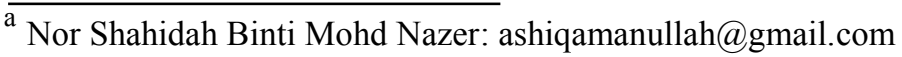


analogue model built upon the combinations of spring and dashpots. In this way, creep and relaxation can be simulated using a single model, i.e. using a single set of parameters, and not treated separately as often happens when empirical models are used. Using a single model to capture both creep and relaxation is important in modelling the kinematics of a landslide since viscous response of clay geo-materials that controls landslide movements is never purely 'creep' mode [9]. Experimental tests to gain insight into viscous responses in shear were carried out under both saturated and unsaturated conditions.

\section{Experimental Criteria}

\subsection{Sample preparation}

\subsubsection{Saturated samples}

Samples were prepared from slurry by mixing Ball clay powder with demineralised water to achieve $100 \%$ water content (about $1.5 \mathrm{w}_{1}$ ). The slurry was placed in the 60 $\mathrm{mm}$ side shearbox and initially let to consolidate under the weight of the loading cap $(\sim 2.7 \mathrm{kPa})$ for 1 day. To ensure that the sample remains saturated, the shear box container was completely filled with water. Afterwards, samples were consolidated in steps to $100 \mathrm{kPa}$ normal stress. Tests were performed in a temperature-controlled room.

Saturated samples in the residual state were prepared by 8 reverse shearing $(8 \mathrm{~mm}$ displacement for each stroke). This was sufficient to ensure the attainment of residual state

\subsubsection{Unsaturated samples}

Unsaturated samples were prepared by consolidating the slurry in the same manner but without the presence of water in the shear box container. Once done, samples were removed from the shear box frame and air-dried to a target water content estimated by weighing the samples. Prior to cutting and trimming, samples were then wrapped with parafilm and sealed in polythene bag to allow moisture equalization. The samples were cut and trimmed using a $60 \mathrm{~mm}$ x $60 \mathrm{~mm}$ square cutter. The cutter was advanced slowly into the sample using a clamp. Since the samples were stiff, cutting could be achieved with ease.

Prior to moving the specimen into the shear box, silicon grease was placed between the bottom plate and the lower frame and between the lower and upper frames of the shearbox. The specimen was then transferred into the $60 \mathrm{~mm}$ side shearbox

The loading cap was put in place and parafilm tape was used to cover the gap between the loading cap and the upper frame) in order to prevent evaporation of the sample. The silicone grease and the parafilm tape were aimed at ensuring constant the water constant throughout the test. The specimen was then ready for the shearing test.

\subsection{Tests for viscous response in shear}

\subsubsection{Displacement -controlled test}

Displacement controlled-test was performed using conventional direct shear box. Shear rate of $0.05 \mathrm{~mm} / \mathrm{min}$ was used in this research. This rate was sufficiently low to ensure drained conditions during shearing. A horizontal displacement was applied until a target shear stress was reached. The horizontal displacement was then maintained constant for approximately one day to allow for the shear stress to 'relax'.

\subsubsection{Stress-controlled test}

Stress controlled-test was performed using a modified direct shear box. The modification involved an additional pulley system which was set in series with the load cell. The tension force applied by the pulley system to the upper frame converted into the shear force applied to the sample. The test was performed by applying the shear force in steps to reach a target shear stress. This was then maintained constant for approximately one day to allow the displacement to 'creep'.

\section{Experimental results and modelling}

\subsection{Viscous response in saturated clay}

\subsubsection{Pre-peak}

Stress relaxation and creep response in saturated soil are shown in Figure 1. Specimens S_50 were sheared to a target shear stress of $27 \mathrm{kPa}$ either by controlling the displacement for stress relaxation test (Figure 1a) or by controlling the shear stress for creep test (Figure 1b). The target shear stress was about $50 \%$ of the ultimate shear strength. To model viscous response of the clay, analogue mechanical models built upon combinations of springs and dashpots, i.e. generalised Kelvin and Maxwell models, were considered. This is because these models allow capturing the creep and relaxation response using a single set of model parameters.

The mechanical model considered is shown in Table 1 (M5). It was selected because of its ability to reproduce stress decay (relaxation) or displacement evolution (creep) that tends to level off. In other words, this model allows reproducing, at least qualitatively, the response observed experimentally. Model parameters were determined by the least-square method to minimise errors between experimental data and simulation for both tests simultaneously.

Because the loading system is not infinitely stiff, another spring was added in series to the model M5 to take into account the compliance of the system. The stiffness of the system was inferred from a combination of tests on dummy samples and the defamation of the system measured during relaxation.

The performance of the M5 model in simulating the relaxation response (Figure 1a) and the creep response 
(Figure 1b) can be considered satisfactory considering that the model only includes a single dashpot. A more accurate simulation of the time-dependent response could be achieved by adding another dashpot into the mechanical model. It is worth noticing that a single set of parameters for the M5 model appears to satisfactorily

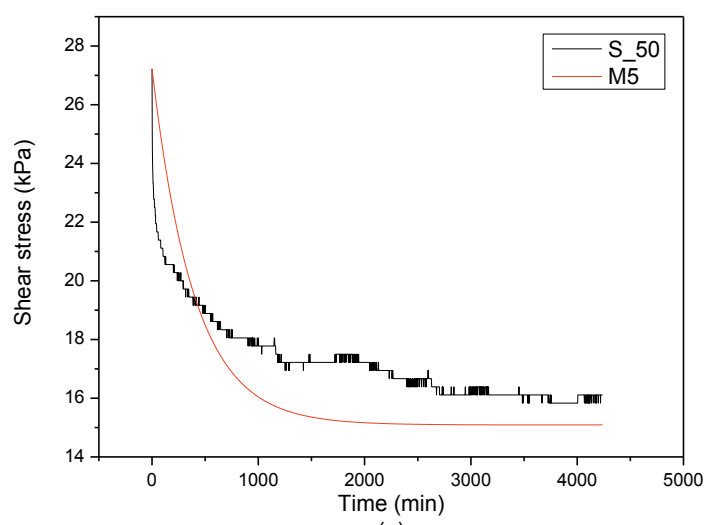

(a)

Figure 1. Viscous responses in saturated soil and simulation curve from M5 and M6 model: (a) stress relaxation (b) creep

Table 1. Creep and relaxation responses for various viscoelastic models after [10]

\begin{tabular}{lcccc}
\hline MODEL & NAME & CONSTITUTIVE EQUATION & CREEP COMPLIANCE, $J(t)$ & RELAXATION MODULUS, G(t) \\
\hline M5 & $\begin{array}{l}G_{1} G_{2} \gamma+G_{1} \eta_{2} \dot{\gamma}= \\
\left(G_{1}+G_{2}\right) \sigma+\eta_{2} \dot{\sigma}\end{array}$ & $\frac{e^{-t / \tau}}{G_{1}}+\frac{G_{1}+G_{2}}{G_{1} G_{2}}\left(1-e^{-1 / \tau_{2}}\right)$, & $G_{1} e^{-t / \tau^{\prime}}+\frac{G_{1} G_{2}}{G_{1}+G_{2}}\left(1-e^{-1 / \tau^{2}}\right)$, \\
& & or $\frac{1}{G_{1}}+\frac{1}{G_{2}}\left(1-e^{-1 / \tau_{2}}\right)$ & or $\frac{G_{1}}{G_{1}+G_{2}}\left(G_{2}+G_{1} e^{-1 / \tau^{\prime}}\right)$ \\
& & & where $\tau^{\prime}=\frac{\eta_{2}}{G_{1}+G_{2}}$
\end{tabular}

\subsubsection{Residual}

Figure 2 shows the viscous response in saturated soil at the residual (post-peak) state. Specimens R 60 were sheared to a target shear stress of $30 \mathrm{kPa}$, equivalent to $60 \%$ of ultimate shear strength. Stress relaxation response (Figure 2a) and creep response (Figure $2 b$ ) are presented herein. Using the same set of parameters applied in the capture the final viscous response in both relaxation and creep mode. Overall, it appears that the M5 model able to reproduce both types of response, creep and relaxation respectively, using only a single set of parameters.

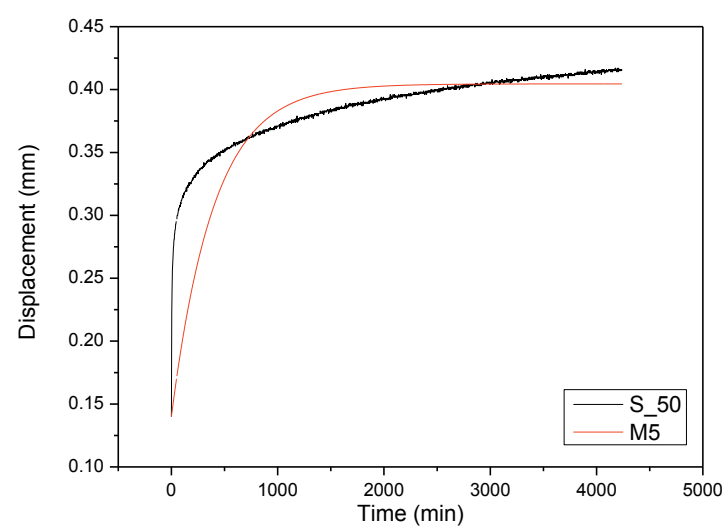

(b) 


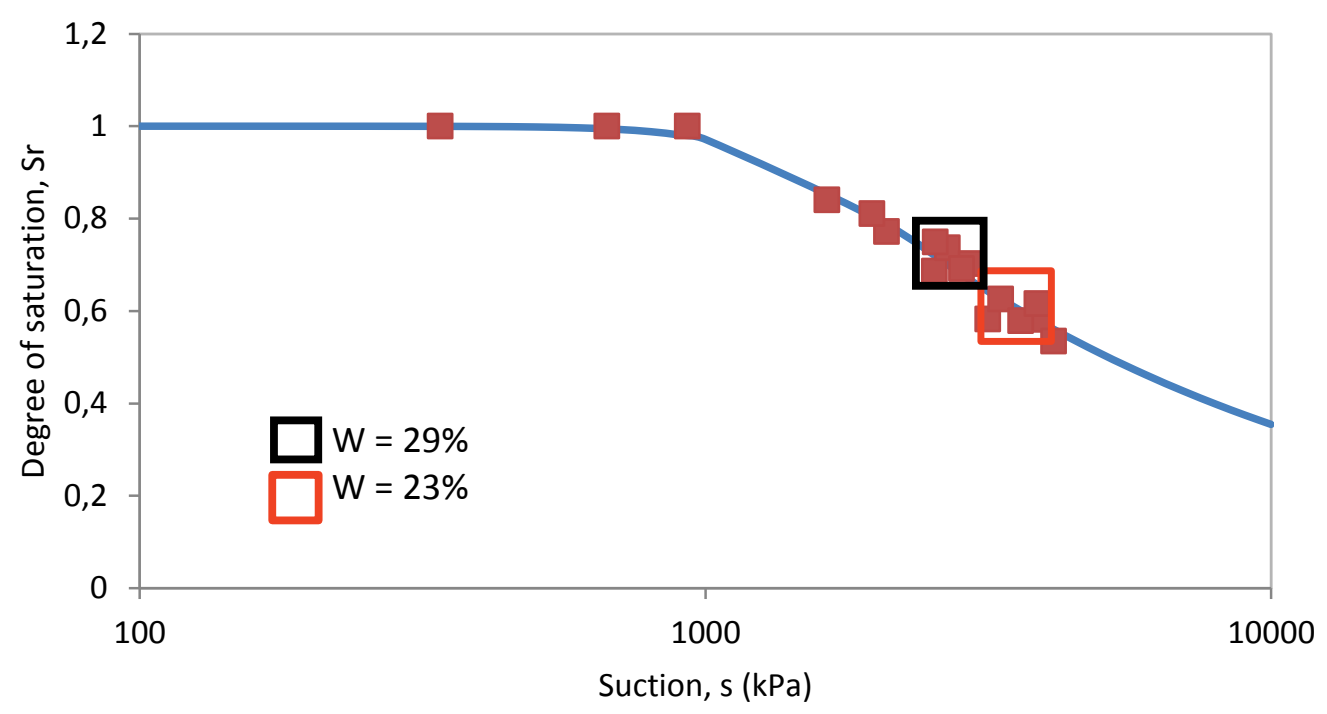

Figure 3. Water retention curve for ball clay

\subsection{Viscous response in unsaturated clay}

The water retention curve of the Ball clay is shown in Figure 3. The air entry value was found to be around $1000 \mathrm{kPa}$. Two tests at different water content $(23 \%$ and $29 \%$ ) were performed. The degree of saturation of these two specimens is shown in Figure 3, and these were equal to 0.5 and 0.75 for the specimens having water content of $23 \%$ and $29 \%$ respectively.

Differences in water content values before and after shearing were observed suggesting that some evaporation did take place throughout the shearing and viscous stages (Table 2). Although the corresponding change in suction was not very significant, it clearly appeared that the antievaporation system should be improved.

Stress relaxation and creep response in unsaturated soil are shown in Figure 4. Specimen US-23_30 refers to unsaturated soil sheared at water content $23 \%$ and to a target shear stress of $78 \mathrm{kPa}$, equivalent to $30 \%$ of ultimate shear strength. Similarly, US-29_30 refers to a specimen sheared at $29 \%$ water content and to a target shear stress of $76 \mathrm{kPa}$, corresponding to $30 \%$ of ultimate shear strength.

Viscous responses in unsaturated soils were showing similar curve trend as in saturated soils and, from these preliminary observations, viscous responses in unsaturated soil does not seem to vary too much with the degree of saturation.

Simulation of M5 model, based on the parameters derived from the tests on saturated samples in the prepeak stage, seems to overestimate both creep and relaxation response (M5_Sat in Figure 4). However, a more satisfactory simulation can be obtained if the model parameters are revaluated by fitting the experimental data for the unsaturated samples (M5_US in Figure 4).

Again, it appears that the M5 model able to reproduce both types of response, creep and relaxation respectively, using only a single set of parameters (though model parameters appear to be different between saturated and unsaturated states).

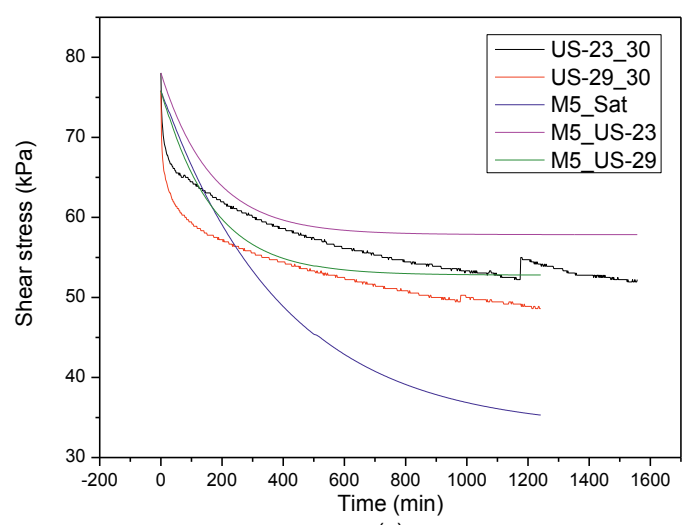

(a)

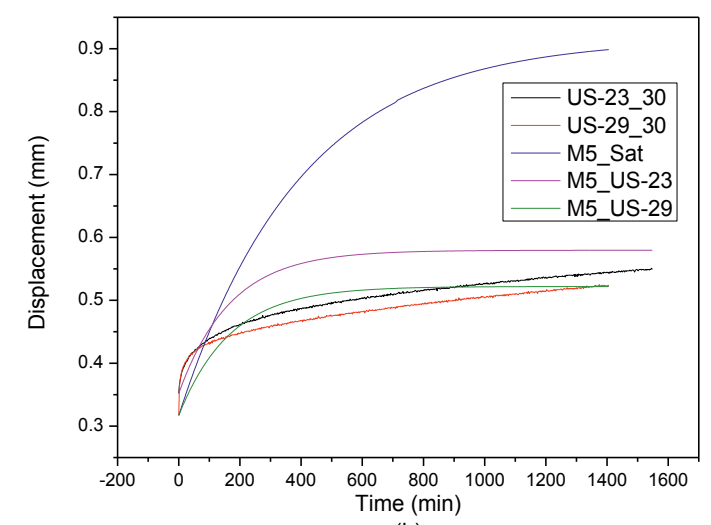

(b)

Figure 4. Viscous responses in unsaturated soil and simulation curves from M5 model: (a) stress relaxation (b) creep. (US=experimental data; M5_Sat=simulation using parameters derived from the pre-peak test; M5_US=simulation based on best-fitting parameters for unsaturated sample). 
Table 2. Matric suction and water content for unsaturated soil

\begin{tabular}{|c|c|c|c|c|c|c|c|c|c|}
\hline \multirow[b]{3}{*}{ Water content } & \multirow[b]{3}{*}{ tau } & \multicolumn{4}{|c|}{ Relaxation } & \multicolumn{4}{|c|}{ Creep } \\
\hline & & \multicolumn{2}{|c|}{ Before } & \multicolumn{2}{|c|}{ After } & \multicolumn{2}{|c|}{ Before } & \multicolumn{2}{|c|}{ After } \\
\hline & & Water content & suction (MPa) & Water content & suction (MPa) & Water content & suction (MPa) & Water content & suction $(\mathrm{MPa})$ \\
\hline 0.29 & $30 \%$ & 25.98 & -2.68 & 24.75 & -2.95 & 26.41 & -2.55 & 24.37 & -2.84 \\
\hline 0.23 & $30 \%$ & 22.07 & -3.33 & 20.42 & -3.61 & 21.72 & -3.86 & 18.86 & -4.13 \\
\hline
\end{tabular}

\section{Conclusions}

The paper has presented experimental data on the viscous response of clay in different states (pre-peak state under saturated conditions, residual state under saturated conditions, and pre-peak state under unsaturated conditions). The viscous response has been investigated in terms of both creep and relaxation on samples starting from the same initial state. A mechanical analogue viscous model (M5) has been used to attempt modelling creep and relaxation simultaneously.

For samples tested in the pre-peak state, the model selected (M5) has proven successful in modelling creep and relaxation response with a single set of parameters. However, the model parameters appear to change from saturated to unsaturated states. In particular, the partial saturation appears to dampen the viscous response in terms of both creep and relaxation.

The residual state appears more difficult to model with a single set of parameters. The previous loading history (reverse shearing) appears to have a different effect on creep and relaxation.

\section{References}

1. E. Intrieri, G. Gigli, N. Casagli, F. Nadim, Brief communication "Landslide Early Warning System: toolbox and general concepts", Nat. Hazards Earth Syst. Sci. 13, 85-90 (2013)

2. L. Picarelli, G. Urciuoli, C. Russo, Effect of groundwater regime on the behaviour of clayey slopes. Can. Geotech. J. 41(3), 467-484 (2004).
3. W. H. Schulz, J. P. McKenna, J. D. Kibler, g. Biavati, Relations between hydrology and velocity of a continuously moving landslide-evidence of porepressure feedback regulating landslide motion? Landslides, 6(3), 181-190 (2009)

4. S. Bernardie, N. Desramaut, J. P. Malet, M. Gourlay, G. Grandjean, Prediction of changes in landslide rates induced by rainfall. Landslides, 1-14 (2014)

5. G. Capparelli, P. Versace, Analysis of landslide triggering conditions in the Sarno area using a physically based model. Hydrol Earth Syst Sci. 18, 3225-3237 (2014)

6. G. Furuya, K. Sassa, H. Hiura, H. Fukuoka, Mechanism of creep movement caused by landslide activity and underground erosion in crystalline schist, Shikoku Island, southwestern Japan. Engineering geology, 53(3), 311-325 (1999).

7. W. Huang, D. Y. Liu, B. Y. Zhao, Y. B. Feng, Y. C. Xia, Study on the Rheological Properties and Constitutive Model of Shenzhen Mucky Soft Soil. J . Eng. Sci. Technol. Rev, 7(3), 55-61. (2014)

8. X. L. Lai, S. M. Wang, W. M. Ye, Y. J. Cui, Experimental investigation on the creep behavior of an unsaturated clay. Can. Geotech. J. 51(6): 621-628 (2014)

9. R. I. Borja, Generalized creep and stress relaxation model for clays, J. Geotech. Eng, 118(11), 17651786 (1992)

10. Continuum Mechanics for Engineers, Second Edition. (1999) 